\title{
Shutterless ion mobility spectrometer with fast pulsed electron source
}

Cite as: Rev. Sci. Instrum. 88, 024102 (2017); https://doi.org/10.1063/1.4976021

Submitted: 16 December 2016 . Accepted: 29 January 2017 . Published Online: 13 February 2017

E. Bunert (D), A. Heptner, T. Reinecke, A. T. Kirk (D), and S. Zimmermann
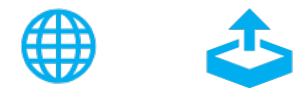

\section{ARTICLES YOU MAY BE INTERESTED IN}

\section{A compact high-resolution X-ray ion mobility spectrometer}

Review of Scientific Instruments 87, 053120 (2016); https://doi.org/10.1063/1.4950866

Fast pulsed operation of a small non-radioactive electron source with continuous emission current control

Review of Scientific Instruments 86, 065102 (2015); https://doi.org/10.1063/1.4921707

High sensitivity field asymmetric ion mobility spectrometer

Review of Scientific Instruments 88, 035115 (2017); https://doi.org/10.1063/1.4978960

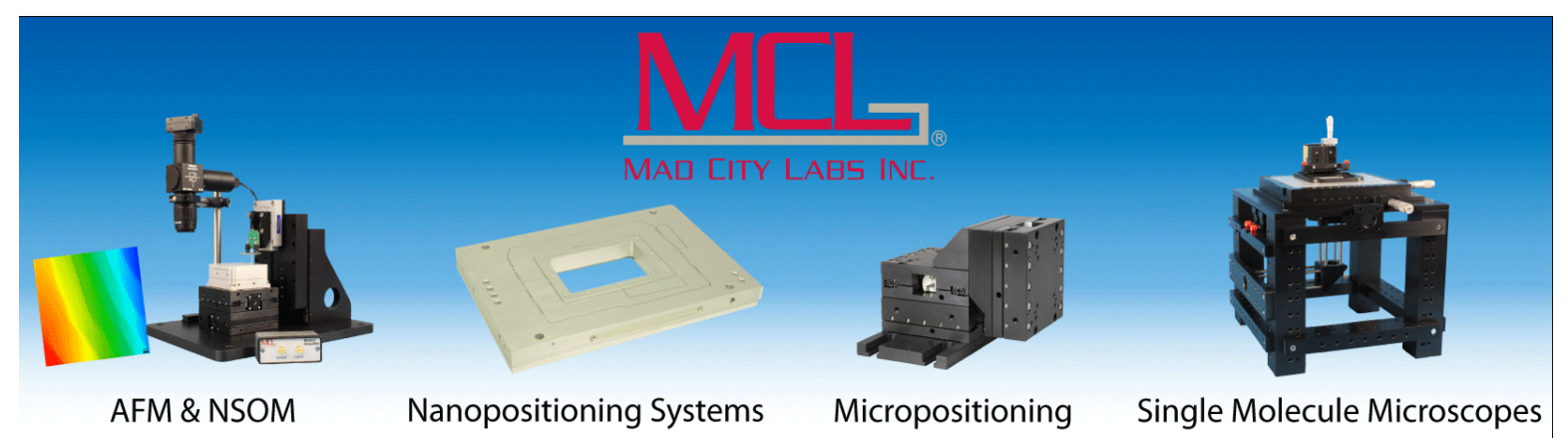




\title{
Shutterless ion mobility spectrometer with fast pulsed electron source
}

\author{
E. Bunert, A. Heptner, T. Reinecke, A. T. Kirk, and S. Zimmermann \\ Department of Sensors and Measurement Technology, Institute of Electrical Engineering and Measurement \\ Technology, Leibniz Universität Hannover, Appelstr. 9a, 30167 Hannover, Germany
}

(Received 16 December 2016; accepted 29 January 2017; published online 13 February 2017)

\begin{abstract}
Ion mobility spectrometers (IMS) are devices for fast and very sensitive trace gas analysis. The measuring principle is based on an initial ionization process of the target analyte. Most IMS employ radioactive electron sources, such as ${ }^{63} \mathrm{Ni}$ or ${ }^{3} \mathrm{H}$. These radioactive materials have the disadvantage of legal restrictions and the electron emission has a predetermined intensity and cannot be controlled or disabled. In this work, we replaced the ${ }^{3} \mathrm{H}$ source of our IMS with $100 \mathrm{~mm}$ drift tube length with our nonradioactive electron source, which generates comparable spectra to the ${ }^{3} \mathrm{H}$ source. An advantage of our emission current controlled nonradioactive electron source is that it can operate in a fast pulsed mode with high electron intensities. By optimizing the geometric parameters and developing fast control electronics, we can achieve very short electron emission pulses for ionization with high intensities and an adjustable pulse width of down to a few nanoseconds. This results in small ion packets at simultaneously high ion densities, which are subsequently separated in the drift tube. Normally, the required small ion packet is generated by a complex ion shutter mechanism. By omitting the additional reaction chamber, the ion packet can be generated directly at the beginning of the drift tube by our pulsed nonradioactive electron source with only slight reduction in resolving power. Thus, the complex and costly shutter mechanism and its electronics can also be omitted, which leads to a simple low-cost IMS-system with a pulsed nonradioactive electron source and a resolving power of 90. Published by AIP Publishing. [http://dx.doi.org/10.1063/1.4976021]
\end{abstract}

\section{INTRODUCTION}

Drift tube ion mobility spectrometers (IMS) can be used for the detection of trace gases by ionizing the analyte and separating the different ion species based on their drift time through a neutral gas under the influence of an electric field. ${ }^{1}$ Commonly, the ionization is initiated by high kinetic energy electrons, emitted from a radioactive beta minus decay, such as from ${ }^{3} \mathrm{H}$ or ${ }^{63} \mathrm{Ni}$. An ion shutter mechanism pushes the generated ions in a small packet into the drift region where the ions are separated. A small ion packet is necessary for the required resolving power and, respectively, the selectivity. For this purpose, a Bradbury-Nielsen shutter is typically used in many applications. ${ }^{1,2}$ Alternatively, we employ a field switching shutter (FS) to realize ion mobility spectrometers with high analytical performance. ${ }^{2}$ However, the disadvantages of radioactive electron sources are legal restrictions, complicating import, export, deployment in certain areas, and disposal of such devices. Furthermore, the electron emission can neither be controlled nor disabled. Therefore, the interest in nonradioactive alternatives has grown in recent years. Possible alternatives are, e.g., UV photo ionization lamps, ${ }^{3,4}$ corona discharge, ${ }^{5-7} \mathrm{X}$-ray sources, ${ }^{8}$ or nonradioactive electron sources. ${ }^{9,10}$ Unfortunately, the generated spectra by UV-photoionization and corona discharge differ from the spectra of a radioactive electron source, so they are not comparable. ${ }^{3,6} \mathrm{X}$-ray sources generate comparable spectra but their disadvantage is a very high penetration depth. Therefore, setup modifications are required to prevent the additional ionization of the drift gas in the drift region. ${ }^{8}$ Nonradioactive electron sources can provide several distinct advantages compared to their radioactive counterparts. No regulatory restrictions exist and the experimental parameters, such as the emitted electron current or the kinetic energy of the electrons, can be controlled.

In addition, our electron source, which is based on thermionic electron emission, can be operated in a fast pulsed mode. ${ }^{9}$ The short electron pulses with high intensity generate small ion packets at simultaneously high ion density, directly at the beginning of the drift region. This can be used for a source controlled injection, as described in Ref. 1, where the required initial small ion packet is generated by the pulsed electron source.

Thus, using a source controlled injection, a complex ion shutter mechanism, including necessary isolated, complex and therefore costly electronics, can be omitted. Although the control electronics of the nonradioactive electron source are still required, the major advantage of this setup is the simplified IMS design combined with less electronics compared to an IMS with a nonradioactive electron source and additional shutter. A first experiment in Ref. 9 has provided the proof of principle of this kind of shutterless IMS. In this work, we focus on the characterization and optimization of a shutterless IMS, via theoretical considerations supported by simulations and verification via measurements.

\section{PRINCIPLE OF IMS AND NONRADIOACTIVE ELECTRON SOURCE}

\section{A. IMS with field switching ion shutter}

Figure 1 shows a schematic of our conventional IMS with our nonradioactive electron source for ionization. The IMS can be separated in three sections: the ionization region between the electron source and the injection grid, the drift region 


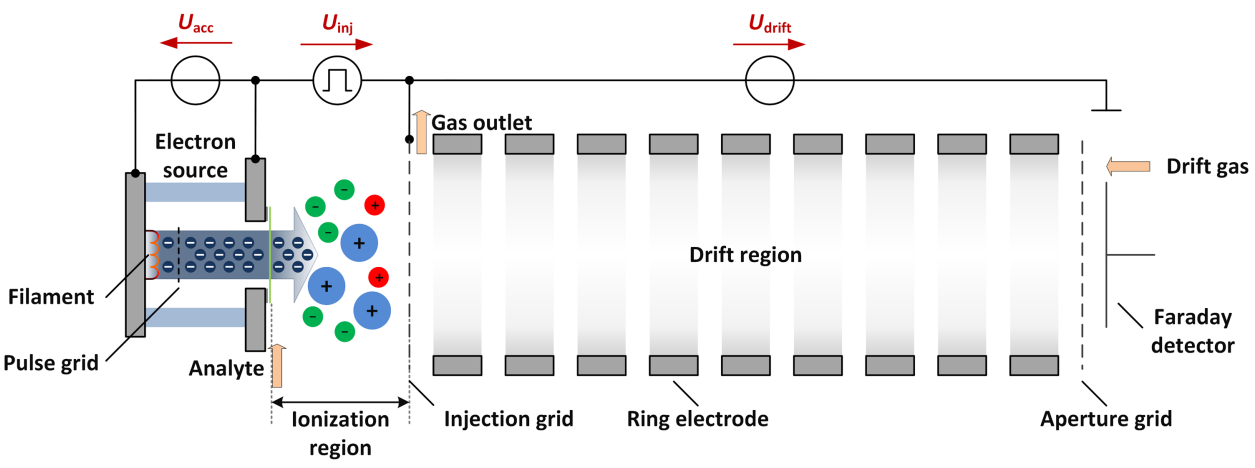

FIG. 1. Schematic of an IMS with field switching shutter and a nonradioactive electron source for ionization. The ionization process takes places in the ionization region. The generated ions can be injected as a small ion packet into the drift region by the injection voltage pulse $U_{\text {inj }}$ with a shutter time of typically $t_{\text {inj }}=20 \mu$ s. between injection and aperture grid, and the Faraday detector. For analyzing trace gases, the analyte is injected into the ionization region. Here, the electron source initiates the atmospheric pressure chemical ionization (APCI) process. In the first step, reactant ions are generated from purified air. Subsequently, these reactant ions ionize the analyte molecules via proton transfer reaction.

Afterwards, the field switching shutter (FS) injects the generated ions into the drift region. The working principle of the FS is described in Ref. 2 and based on switching the injection voltage $U_{\text {inj }}$ and thus the electrical field in the ionization region on and off, while the potential of the injection grid remains constant. During the shutter time of typically $t_{\text {inj }}=20 \mu \mathrm{s}$, a significantly higher potential is applied to the electron source than that of the injection grid. Therefore, the ions are pushed into the drift region as a small ion packet. The advantages of the FS compared to the widely used BradburyNielsen shutter are easier manufacturing of the mechanical parts, improved mechanical stability, and reduced distortion of the drift field. ${ }^{2}$ The latter substantially influences the resolving power in compact drift tubes. To achieve a homogeneous electrical field in the drift region, the drift voltage of $U_{\text {drift }}=5 \mathrm{kV}$ is applied via a resistive voltage divider to stainless steel ring electrodes separated by polyether ether ketone (PEEK) isolation rings. Accelerated by the drift field, the injected ion packet drifts towards the detector and thereby the different ion species are separated by their specific mobility in a neutral drift gas. Finally, the ions discharge on the Faraday detector and the resulting ion current is plotted over the drift time to obtain the ion mobility spectrum. The key for a high analytical performance is an efficient separation of the different ion species. ${ }^{11}$ The resolving power $R$ is a measure for the sharpness of a detected peak, which is described by Eq. (1). ${ }^{1}$ A high resolving power is gained, when the full width at half maximum (FWHM) $w_{0.5}$ of the measured ion peaks is short compared to their drift time $t_{\mathrm{drift}}$,

$$
R=\frac{t_{\mathrm{drift}}}{w_{0.5}}
$$

\section{B. Nonradioactive electron source}

The initial ionization of the analyte has a major impact on the achievable resolving power of a shutterless IMS. To investigate the influence of the ionization process on the generated ion distribution, the employed ionization source, i.e., the nonradioactive electron source, is described in this section. The average kinetic electron energy of a ${ }^{3} \mathrm{H}$ source commonly used in IMS applications is about $E_{\mathrm{kin},{ }^{3} \mathrm{H}}=5.7 \mathrm{keV} .{ }^{12}$ To realize an electron source, generating electrons with comparable kinetic energies, we place a hot filament in a vacuum chamber to generate free electrons by thermionic emission. Subsequently, the electrons extracted from the hot filament are accelerated in a vacuum chamber by an acceleration voltage of up to $U_{\text {acc }}$ $\leq 15 \mathrm{kV}$. At the end of the acceleration region, the vacuum chamber is sealed with a $300 \mathrm{~nm}$ thin $\mathrm{Si}_{3} \mathrm{~N}_{4}$-membrane. Electrons with a kinetic energy of more than $5 \mathrm{keV}$ can partially transmit through the membrane into the ionization region. The relative electron transmission and average kinetic energy of the electrons depend on the acceleration voltage, which is simulated with the Monte Carlo simulation software Casino, and shown in Figure 2.

For acceleration voltages of more than $8 \mathrm{kV}$, approximately $50 \%$ of the accelerated electrons can transmit through the membrane. After transmission, the electrons have an average kinetic energy of more than $4 \mathrm{keV}$. By simply varying the acceleration voltage of our electron source between $8 \mathrm{kV}$ $\leq U_{\text {acc }} \leq 14 \mathrm{kV}$, the average kinetic energy of the emitted electrons can be adjusted. To reduce mechanical stress in the membrane, our electron source typically operates at $U_{\text {acc }}=10 \mathrm{kV}$, leading to emitted electrons with an average kinetic energy of $E_{\text {kin }}=7.2 \mathrm{keV}$, which is comparable to a radioactive ${ }^{3} \mathrm{H}$-source. . $^{9} 10,12,13$ A major advantage of our nonradioactive electron source is that the electron emission current is adjustable from a few tens of picoamperes (which is

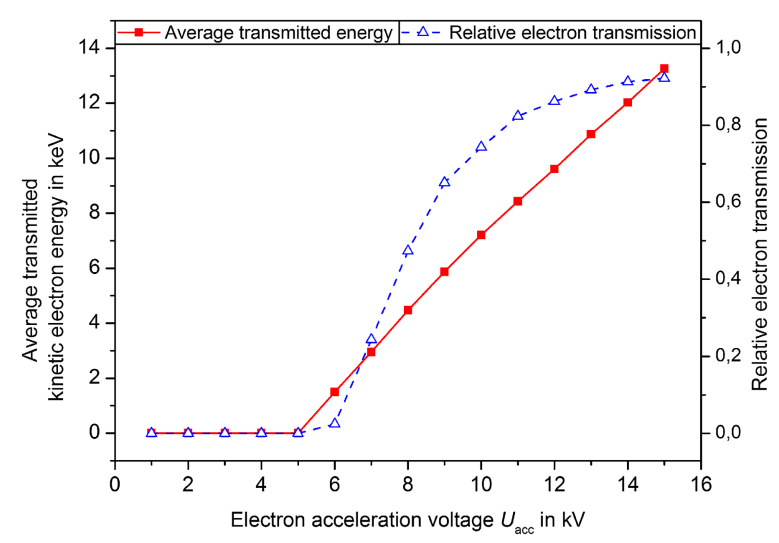

FIG. 2. Simulation results for relative transmission of high kinetic electrons through a $300 \mathrm{~nm}$ thin $\mathrm{Si}_{3} \mathrm{~N}_{4}$-membrane (a) and simulation results for average kinetic electron energy after transmitting through a $300 \mathrm{~nm}$ thin $\mathrm{Si}_{3} \mathrm{~N}_{4}$-membrane (b). 
TABLE I. Electron source operating parameters.

\begin{tabular}{lc}
\hline \hline Parameter & Value \\
\hline $\mathrm{Si}_{3} \mathrm{~N}_{4}$ membrane thickness & $300 \mathrm{~nm}$ \\
$\mathrm{Si}_{3} \mathrm{~N}_{4}$ membrane diameter & $1.5 \mathrm{~mm} \times 1.5 \mathrm{~mm}$ \\
Electron acceleration voltage $U_{\text {acc }}$ & $8 \ldots 14 \mathrm{kV}$ \\
Corresponding kinetic electron energies after & $4.5 \ldots 12 \mathrm{keV}$ \\
transmission through $\mathrm{Si}_{3} \mathrm{~N}_{4}$ membrane & $200 \ldots 270 \mathrm{~mA}$ \\
Filament current & $0.2 \ldots 100 \mathrm{nA}$ \\
Emission current & $-120 \mathrm{~V}$ \\
Grid voltage low & $25 \mathrm{~V}$ \\
Grid voltage high & $100 \mathrm{~ns} \ldots \infty$ \\
Electron pulse width/ionization time $t_{\text {ion }}$ & $2 \cdot 10^{-7} \mathrm{hPa}$ \\
Absolute pressure inside the vacuum chamber & \\
\hline \hline
\end{tabular}

comparable to the electron emission of a tritium source with $300 \mathrm{MBq}$ ) up to hundreds of nanoamperes.

To operate the electron emitter in pulsed mode, ${ }^{9}$ we place a grid in front of the hot filament for switching the electron beam passing through the silicon nitride membrane. By optimizing the geometric parameters and the control electronics, we can achieve electron pulses with a definable pulse width of down to a few nanoseconds, whereby the emission current can still be adjusted in the range mentioned above. The operating parameters of the electron source used in this work are listed in Table I.

\section{Shutterless IMS with pulsed electron source}

The design of the shutterless IMS is similar to our conventional IMS design. The biggest difference is that there is no restricted ionization region anymore. This leads to a combined drift and ionization region where the ionization takes place at the beginning of the drift region, as shown in Figure 3. The second part of the drift region is the actual drift region, where the generated ion species are separated. For a modular experimental setup, the electrical fields in the ionization region $E_{\text {ion }}$ and in the actual drift region $E_{\text {drift }}$ are adjustable by two independent voltage sources $U_{\mathrm{E} \text {,ion }}$ and $U_{\text {drift }}$ (Figure 3 ).

The drift tube used in this work is based on the drift tube described in Refs. 14 and 15 with a drift length of $l_{\text {drift }}$ $=100 \mathrm{~mm}$. The IMS operating parameters are listed in Table II.

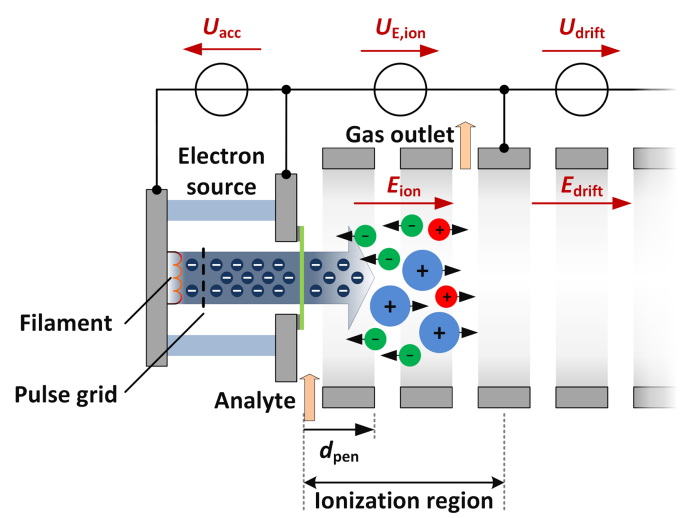

FIG. 3. Ionization scheme of a shutterless IMS with pulsed electron source. The short electron pulse generates a small ion packet in the ionization region as a part of the drift region. The generated ions are subsequently accelerated by the adjustable electrical field $E_{\text {ion }}$ towards the detector.
TABLE II. IMS operating parameters.

\begin{tabular}{lc}
\hline \hline Parameter & Value \\
\hline Drift length $l_{\text {drift }}$ & $100 \mathrm{~mm}$ \\
Drift region diameter & $15 \mathrm{~mm}$ \\
Repetition rate & $40 \mathrm{~Hz}$ \\
Drift voltage $U_{\text {drift }}$ & $5 \mathrm{kV}$ \\
Aperture voltage & $100 \mathrm{~V}$ \\
Electrical field in the drift region $E_{\mathrm{drift}}$ & $50 \ldots 100 \mathrm{~V} / \mathrm{mm}$ \\
Electrical field in the ionization region $E_{\mathrm{ion}}$ & $100 \ldots 2500 \mathrm{~V} / \mathrm{mm}$ \\
Drift gas flow & $160 \mathrm{mls} / \mathrm{min}$ \\
Sample gas flow & $10 \mathrm{mls} / \mathrm{min}$ \\
Dew point drift gas and sample carrier & $-89^{\circ} \mathrm{C}$ \\
Operating pressure & $1018 \mathrm{mbar}$ \\
Operating temperature & $25{ }^{\circ} \mathrm{C}$ \\
\hline \hline
\end{tabular}

Note that the IMS in Refs. 14 and 15 use significant different parameters compared to the IMS described in this work (e.g., a higher drift voltage). Therefore, the analytical performance of those setups is not directly comparable. The electrical field of the ionization region is limited to the first $3 \mathrm{~mm}$ of the drift region. The control electronics for the shutterless IMS and the electron source as well as the data recording electronics and software are self-developed for this application. Only the drift voltage $U_{\text {drift }}$ is generated by a power supply from FuG. For the drift and sample carrier gas, purified and dried air is used.

\section{ION GENERATION}

\section{A. Electron penetration depth}

As the ionization region is located directly at the beginning of the drift tube, the ions are generated in a region with a constant electrical field $E_{\text {ion }}$. Therefore, the ions are directly accelerated towards the detector (ions with opposite polarity are accelerated towards the electron source, respectively). Regarding the resolving power of the IMS, described by Eq. (1), there are two major influences on the width of the initial ion packet. As the ions are immediately accelerated towards the detector, the first major influence is the ionization time, which directly adds to the ion packet width in the time domain. Second, the minimum spatial width of the initial ion packet is determined by the penetration depth $d_{\text {pen }}$ of the electrons emitted into the ionization region.

It is obvious that the penetration depth directly depends on the kinetic energy $E_{\text {kin,el }}$ of the emitted electrons and thus on the electron acceleration voltage $U_{\text {acc. }}$. The resulting penetration depth $d_{\text {pen }}$ of the electrons into dry air and the energy deposition in the ionization region are simulated with Casino as function of the kinetic electron energy. Approximately, the deposited energy is proportional to the ion production rate. Plotting the simulation results over the penetration depth, the generated ion densities are illustrated as a function of the distance to the electron source in Figure 4 for three exemplary acceleration voltages $U_{\text {acc }}$.

The simulation results show that both the ion density and the spatial width of the ion distribution increase towards higher kinetic electron energies. This results in a wider 


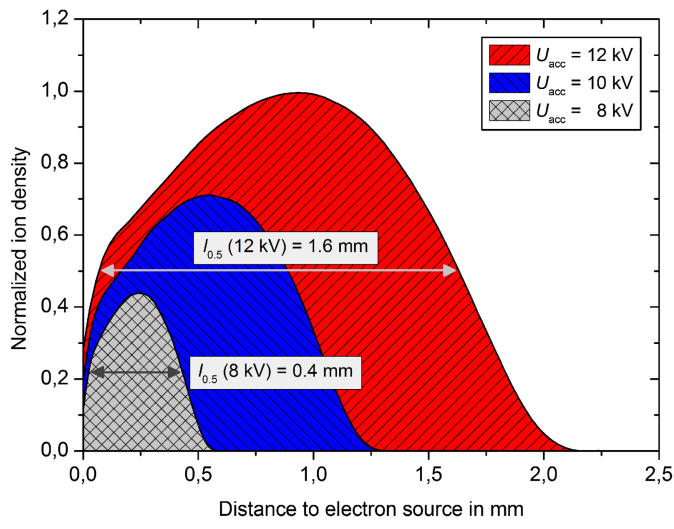

FIG. 4. Simulated normalized ion density as a function of the distance to the electron source for electron acceleration voltages of $8 \mathrm{kV}, 10 \mathrm{kV}$, and $12 \mathrm{kV}$.

ionization region and thus in broadened initial ion packets. While higher ion concentrations improve the sensitivity, wider spatial widths reduce the resolving power of an IMS. Therefore, a compromise must be achieved between high sensitivity and high resolving power by optimizing the parameters.

To investigate the influence of the acceleration voltage on the generated ion distribution, the positive reactant ion peak $\left(\mathrm{RIP}^{+}\right)$, generated by ionizing purified dry air, is measured for different acceleration voltages between $8 \mathrm{kV}$ and $14 \mathrm{kV}$. Hereby, the ionization pulse width is set to a constant value of $t_{\text {ion }}=10 \mu \mathrm{s}$. Furthermore, the emission current (i.e., intensity) of the electron source is adjusted for each acceleration voltage to achieve a constant RIP $^{+}$amplitude of $100 \mathrm{pA}$. The electrical field in both the drift region and the ionization region is set to $E_{\text {ion }}=E_{\text {drift }}=50 \mathrm{~V} / \mathrm{mm}$. Figure 5 shows three examples for the resulting $\mathrm{RIP}^{+}$for $8 \mathrm{kV}, 10 \mathrm{kV}$, and $12 \mathrm{kV}$ acceleration voltages.

As can be expected from the simulation results in Figure 4, the center of the detected $\mathrm{RIP}^{+}$shifts to shorter drift times with increasing acceleration voltages. Furthermore, the FWHM $w_{0.5}$ of the detected $\mathrm{RIP}^{+}$increases with higher kinetic electron energies from $158 \mu \mathrm{s}$ at $U_{\mathrm{acc}}=8 \mathrm{kV}$ to $183 \mu \mathrm{s}$ at $U_{\mathrm{acc}}=12 \mathrm{kV}$ because of the higher penetration depth of the electrons. It is noticeable that the spatial FWHM ratio between the simulated distributions at $U_{\text {acc }}=12 \mathrm{kV}$ and $U_{\text {acc }}=8 \mathrm{kV}$ is 4 , but

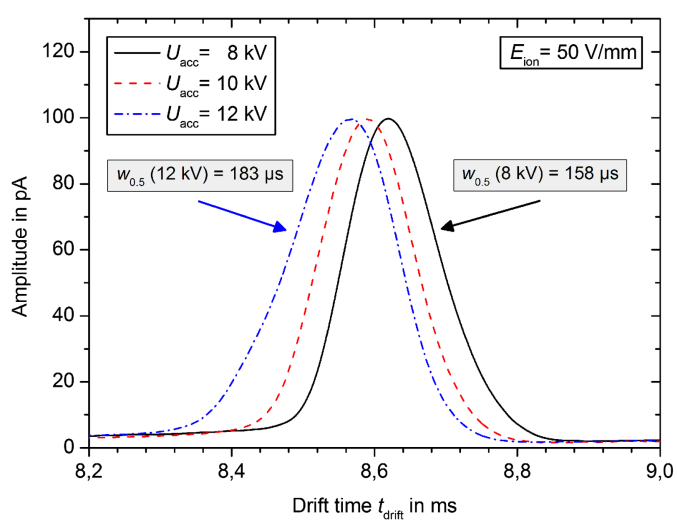

FIG. 5. Broadening of the detected $\mathrm{RIP}^{+}$with increased acceleration voltage and thus increased penetration depth of the electrons. The electrical field in the ionization region and the drift region is $E_{\text {ion }}=E_{\text {drift }}=50 \mathrm{~V} / \mathrm{mm}$, the ionization time is set to $10 \mu \mathrm{s}$. the temporal FWHM ratio of the detected $\mathrm{RIP}^{+} \mathrm{s}$ is only about 1.16. This can be explained by broadening influences during the drift towards the detector, such as diffusional broadening or Coulombic repulsion. Therefore, a detailed investigation is performed in Sec. III B to describe the relations between the initial ion packet and the detected peaks.

\section{B. Initial ion packet width and maximum attainable resolving power}

In this section, the influence of the initial spatial width on the temporal FWHM $w_{0.5}$ of the $\mathrm{RIP}^{+}$and thus on the resolving power is investigated. Therefore, the resulting initial FWHM $w_{\text {init }}$ in the time domain is calculated with Eq. (2). The spatial FWHM $l_{0.5}$ for various acceleration voltages is derived from the ion distribution depicted in Figure 4. Furthermore, the drift velocity $v$ of the ions is required. It is found by multiplication of the ion mobility $K$ of an ion species and the electrical field $E$. As a result of numerous measurements, we specified the ion mobility of the positive reactant ions to $K_{\mathrm{RIP}}=2.31 \mathrm{~cm}^{2} / \mathrm{Vs}$ at $25^{\circ} \mathrm{C}$,

$$
w_{\text {init }}=\frac{l_{0.5}}{v}=\frac{l_{0.5}}{K \cdot E} .
$$

Figure 6 depicts the initial ion FWHM $w_{\text {init }}$, derived from Eq. (2) for various electrical fields $E_{\text {ion }}$ and acceleration voltages $U_{\text {acc. }}$ These results illustrate the relevance of the electrical field $E_{\text {ion }}$ in the ionization region for the initial time domain FWHM $w_{\text {init }}$, particularly at high acceleration voltages and high penetration depths, respectively. The results show that the combination of the lowest acceleration voltage and highest electrical field leads to the smallest $w_{\text {init }}$ and thus to the highest possible resolving power of the IMS.

For calculating the resulting FWHM of the reactant ion peak $\left(\mathrm{RIP}^{+}\right) w_{0.5}$, the relation between the initial FWHM $w_{\text {init }}$ and $w_{0.5}$ can be approached by Eq. (3) as a geometrical addition of the initial width of the ion packet $w_{\text {init }}$, additional broadening influence of the amplifier $w_{\text {amp }}$, the diffusional broadening $w_{\text {diff }}$, and the Coulombic broadening $w_{\text {coul }}:{ }^{11,14,16}$

$$
w_{0.5}=\sqrt{w_{\text {init }}^{2}+w_{\text {diff }}^{2}+w_{\text {coul }}^{2}+w_{\text {amp }}^{2}} .
$$

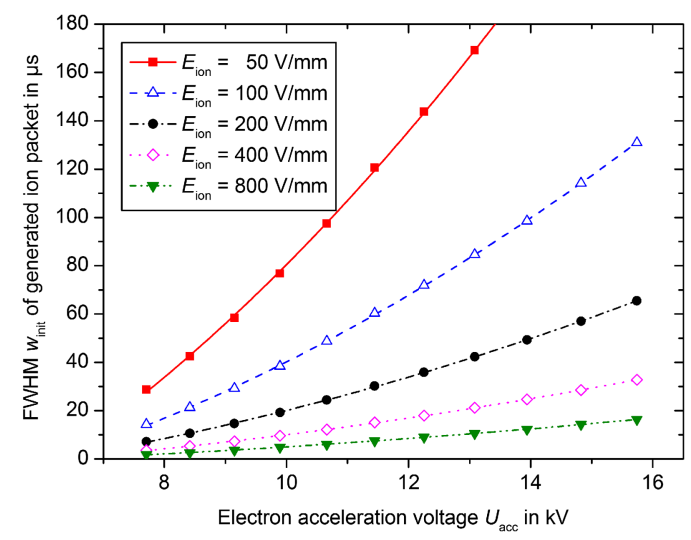

FIG. 6. Resulting initial FWHM $w_{\text {init }}$ in the time domain, based on Figure 4, as a function of the acceleration voltage $U_{\text {acc }}$ for various electrical fields $E_{\text {ion }}$ in the ionization region. The associated ion mobility for the reactant ions at room temperature is $\mathrm{K}_{\mathrm{RIP}}=2.31 \mathrm{~cm}^{2} / \mathrm{Vs}$. 


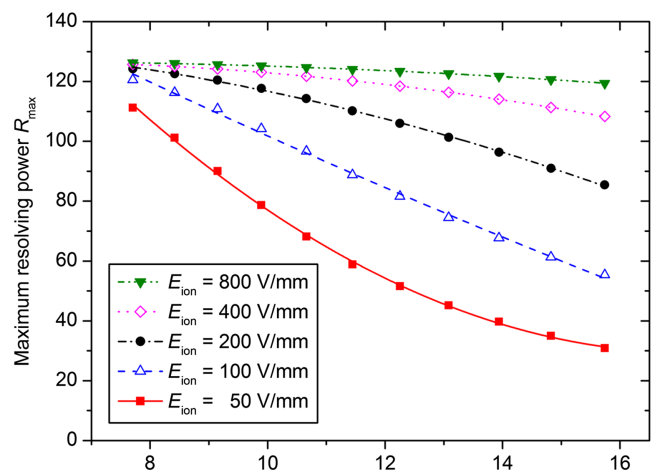

(a)

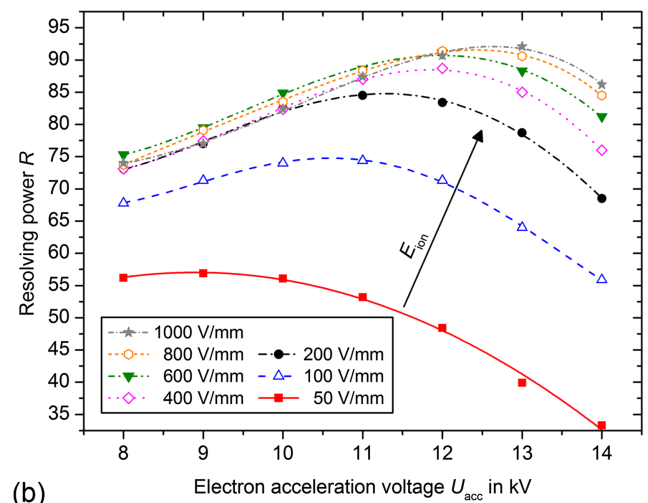

(b)

FIG. 7. Calculated (a) and measured (b) maximum resolving power (of the $\mathrm{RIP}^{+}$) as a function of the acceleration voltage $U_{\text {acc }}$ and the electrical field $\mathrm{E}_{\text {ion }}$, in the ionization region based on the resulting initial FWHM $w_{\text {init }}$ in the time domain of Figure 6 and Eq. (6). The used parameters are drift voltage $U_{\text {drift }}=5 \mathrm{kV}$, drift length $l_{\text {drift }}=100 \mathrm{~mm}$, ion mobility $\mathrm{K}_{\mathrm{RIP}}=2.31 \mathrm{~cm}^{2} / \mathrm{Vs}$, temperature $\mathrm{T}=298.15 \mathrm{~K}$, ionization time $t_{\text {ion }}=10 \mu$ s. The RIP + amplitude is controlled to a constant value of $100 \mathrm{pA}$.

For low ion densities, the Coulombic broadening term $w_{\text {coul }}$ can be neglected, as it is relatively small and the resulting FWHM is dominated by the remaining broadening terms. The influence of the amplifier previously was determined to $w_{\text {amp }}$ $=17 \mu \mathrm{s} .{ }^{15}$ The diffusional broadening $w_{\text {diff }}$ is described by Eq. (4) as a function of the drift voltage $U_{\text {drift }}$ and the drift time $t_{\text {drift }}{ }^{11,17,18}$

$$
w_{\text {diff }}=\left(\frac{16 \mathrm{k}_{\mathrm{B}} T \ln 2}{\mathrm{e} U_{\mathrm{drift}}}\right)^{\frac{1}{2}} t_{\mathrm{drift}} .
$$

The injection width of the initial ion packet for this IMS configuration but with a field switching ion shutter is specified in Ref. 15 as $w_{\text {init }}=5 \ldots 20 \mu$ s. Therefore, we set the electron pulse width to $t_{\text {ion }}=10 \mu$ s for the first investigation of the shutterless configuration. As mentioned above, this time directly adds to the initial time domain FWHM $w_{\text {init }}$ because of the immediate acceleration of the generated ions in the shutterless ionization region. This leads to an extended formulation of Eq. (3) for the minimum FWHM of the measured $\mathrm{RIP}^{+} w_{0.5}$,

$$
w_{0.5}=\sqrt{\left(w_{\text {init }}+t_{\text {ion }}\right)^{2}+w_{\text {diff }}^{2}+w_{\text {amp }}^{2}} .
$$

Insertion of Eqs. (2), (4) and (5) in Eq. (1) yields the following expression for the maximum attainable resolving power $R_{\max }$ :

$$
\begin{aligned}
R_{\max } & \leq \frac{t_{\text {drift }}}{w_{0.5}} \\
& =\frac{\frac{l_{\text {drift }}{ }^{2}}{K \cdot U_{\text {drift }}}}{\sqrt{\left(\frac{l_{0.5}}{K \cdot E_{\text {ion }}}+t_{\text {ion }}\right)^{2}+\frac{16 \mathrm{k}_{\mathrm{B}} T \ln 2}{\mathrm{e} \cdot U_{\text {drift }}} \cdot\left(\frac{l_{\text {drift }}{ }^{2}}{K \cdot U_{\text {drift }}}\right)^{2}+w_{\text {amp }}{ }^{2}}} .
\end{aligned}
$$

With Eq. (6) and the results of Figure 6, we can calculate the maximum resolving power as a function of the acceleration voltage $U_{\text {acc }}$ and the electrical field $E_{\text {ion }}$ in the ionization region, which is plotted in Figure 7(a). The related parameters are listed in the caption. These results indicate that the highest possible resolving power requires a low acceleration voltage and a high electrical field in the ionization region.

Figure 7(b) shows the measuring results corresponding to the theoretical considerations in Figure 7(a). Here, the resolving power of the measured $\mathrm{RIP}^{+}$is plotted over the acceleration voltage for various electrical fields in the ionization region. The measurement results show that there is a maximum resolving power for each electrical field. However, in contrast to the calculation results, the maximum is not found at the lowest acceleration voltage. For example, the plot for an electrical field of $E_{\text {ion }}=100 \mathrm{~V} / \mathrm{mm}$ shows a local maximum at $U_{\text {acc }}$ $=10.6 \mathrm{kV}$ with a resolving power of $R=74.8$. The deviation between calculation and measurement can be explained by the negligence of the Coulomb broadening $w_{\text {coul }}$. For lower acceleration voltages, the intensity of the electron source has to be dramatically increased, in order to generate $\mathrm{RIP}^{+}$amplitudes equal to the amplitudes for high acceleration voltages at the same ionization time. Therefore, Coulombic repulsion increases because of the increased ion density and becomes the major broadening influence on $w_{0.5}$ (see Eq. (3)) and hence on the resulting resolving power. Thus, the resolving power is not a constantly increasing towards lower penetration depths, as suggested by the simulation results in Figure 6. Instead, the resolving power shows a maximum value at a certain acceleration voltage for each electrical field in the ionization region. These voltages and their corresponding electrical field $E_{\text {ion }}$ and resolving power $R$ are shown in Table III.

\section{Ionization time}

The preceding measurements were carried out with an ionization time of $t_{\text {ion }}=10 \mu \mathrm{s}$. As this time directly adds to the initial width $w_{\text {init }}$ of the ion packet, the influence of $t_{\text {ion }}$ on the resolving power is investigated in this section. Hereby, the electrical field $E_{\text {ion }}$ in the ionization region is set to different values between $50 \mathrm{~V} / \mathrm{mm}$ and $1000 \mathrm{~V} / \mathrm{mm}$, while the

TABLE III. Optimum acceleration voltages $U_{\text {acc,opt }}$ at different electrical fields $E_{\text {ion }}$ in the ionization region.

\begin{tabular}{lccccc}
\hline \hline$E_{\text {ion }}$ in V/mm & $U_{\text {acc,opt in kV }} R$ & $E_{\text {ion }}$ in V/mm & $U_{\text {acc,opt in } \mathrm{kV}}$ & $R$ \\
\hline 50 & 9.0 & 56.9 & 600 & 12.0 & 90.7 \\
100 & 10.6 & 74.8 & 800 & 12.4 & 91.6 \\
200 & 11.3 & 84.8 & 1000 & 12.6 & 92.0 \\
400 & 11.8 & 88.5 & & & \\
\hline
\end{tabular}




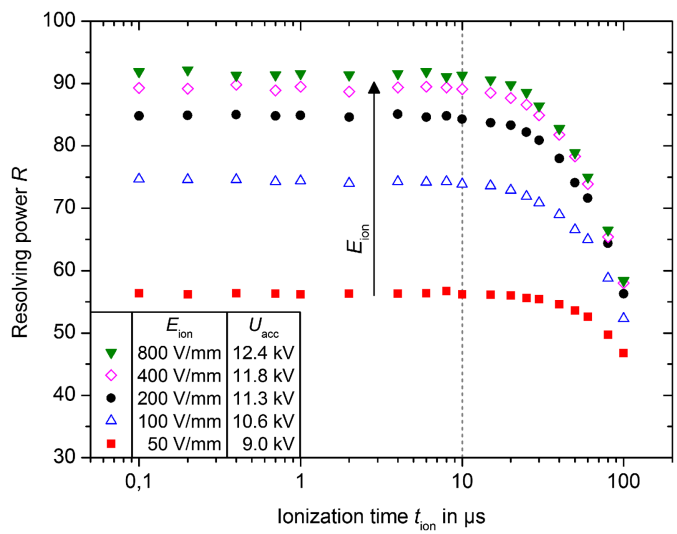

FIG. 8. Resolving power R over ionization time $t_{\text {ion }}$ for different electrical fields $E_{\text {ion }}$ in the ionization region. The acceleration voltage $U_{\text {acc }}$ is always adjusted to the related optimum value, the $\mathrm{RIP}^{+}$amplitude is controlled to a constant value of $100 \mathrm{pA}$, the electrical field in the drift region is $E_{\mathrm{drift}}=50$ $\mathrm{V} / \mathrm{mm}$.

acceleration voltage is adjusted to the previously determined corresponding optimum (see Table III). Figure 8 shows the measured resolving power $R$ of the $\mathrm{RIP}^{+}$with a constant amplitude of $100 \mathrm{pA}$ as a function of the ionization time $t_{\text {ion. }}$. A decreasing resolving power can be observed for ionization times longer than $t_{\text {ion }}>10 \mu$ s because of the direct broadening of the initial ion packet, described in Eqs. (5) and (6), respectively.

However, ionization times shorter than $t_{\text {ion }} \leq 10 \mu$ s do not lead to an improvement of the resolving power. This is attributed to an increased Coulombic repulsion with shorter ionization times, as the intensity of the electron source has to be increased to generate the same quantity of ions to reach equal $\mathrm{RIP}^{+}$amplitudes for all measurements. Furthermore, the influence of the remaining broadening terms dominates the width of the ion packet towards very short ionization times. However, with regard to the reachable minimum limit of detection $(\mathrm{LoD})$ a longer ionization time is favorable. Therefore, the highest possible ionization time without reduction of the resolving power is selected and thus the reaction time for generating the analyte ions is increased.

\section{Limit of detection}

Beside the resolving power, the limit of detection (LoD) of an IMS describes its analytical performance. For a preliminary characterization of our setup, we use acetone as analyte, purchased from Sigma Aldrich. The acetone is filled in a vial heated to $35{ }^{\circ} \mathrm{C}$ in a permeation oven. For generating a constant acetone vapor concentration, the oven is purged with a constant flow of $600 \mathrm{mls} / \mathrm{min}$ of purified dry air. An adjustable fraction of this gas is diluted with dry air for generating different acetone concentrations in the sample gas.

For all measurements, we set the ionization time to $10 \mu \mathrm{s}$. The drift voltage is set to $U_{\text {drift }}=5 \mathrm{kV}$, and the electrical field $E_{\text {ion }}$ in the ionization region is varied between $100 \mathrm{~V} / \mathrm{mm}$ and $800 \mathrm{~V} / \mathrm{mm}$. Hereby, the acceleration voltage is set to its optimum value (see Table III). Furthermore, the RIP $^{+}$amplitude is kept constant at $100 \mathrm{pA}$ without analyte. As the $\mathrm{RIP}^{+}$amplitude
TABLE IV. Total ion charge for different electrical fields $E_{\text {ion }}$ in the ionization region with a constant $\mathrm{RIP}^{+}$amplitude of $100 \mathrm{pA}$, without analyte.

\begin{tabular}{lcccc}
\hline \hline$E_{\text {ion }}$ in V/mm & 100 & 200 & 400 & 800 \\
$Q_{\text {tot }}$ in $\mathrm{fC}$ & 12.3 & 10.6 & 9.9 & 9.6 \\
\hline
\end{tabular}

cannot be used as a measure for the source activity when measuring a gas mixture, the total ion charge $Q_{\text {tot }}$ of the detected spectrum is determined. The results are shown in Table IV.

Adjusting the total ion charge for every $E_{\text {ion }}$ by adjusting the filament current ensures a constant electron emission of the electron source and thus a constant ion quantity for all measurements. Figure 9 shows an exemplary spectrum of the shutterless IMS for an acetone concentration of $20 \mathrm{ppb}_{\mathrm{v}}$. The related parameters are listed in the caption.

Subsequently, we determined the LoD for different $E_{\text {ion }}$ based on the measured noise of the baseline and the measured linear amplitude increase of the acetone monomer peak at low concentrations. The LoD is defined as three times the standard deviation of the noise. The determined LoDs for a measuring time of $1 \mathrm{~s}$ are listed in Table V. The respective resolving power $R$ of the $\mathrm{RIP}^{+}$without analyte in the ionization region is also listed in this table as a reminder. For the maximum reachable resolving power of $R=92$ at $E_{\text {ion }}=800 \mathrm{~V} / \mathrm{mm}$ we achieve a LoD for acetone of about $4 \mathrm{ppb}_{\mathrm{v}}$. This can be slightly improved to $\approx 2 \mathrm{ppb}_{\mathrm{v}}$ by reducing the electrical field to $E_{\mathrm{ion}}$ $=100 \mathrm{~V} / \mathrm{mm}$ and hence increasing the reaction time, but with a slightly decreased resolving power of $R=75$.

For comparison, the corresponding $\mathrm{LoD}_{\mathrm{FS}}$ and resolving power $R_{\mathrm{FS}}$ of this IMS equipped with field switching shutter and an ionization time of $t_{\text {ion }}=10 \mu$ s is about $\mathrm{LoD}_{\mathrm{FS}}=1 \mathrm{ppb}_{\mathrm{v}}$ at $R_{\mathrm{FS}}=92$. Therefore, we reach nearly the same LoD for acetone as with the shutterless IMS at low electrical fields $E_{\text {ion }}$ in the ionization region. The resolving power $R_{\mathrm{FS}}$ is comparable to that one of the shutterless IMS at high $E_{\text {ion }}$. This suggests that we found the parameters for the best achievable LoD at the highest possible resolving power with this shutterless IMS. Depending on the application, the focus can be set on either a low LoD or a high resolving power only by adjusting the electrical field $E_{\text {ion }}$ in the ionization region.

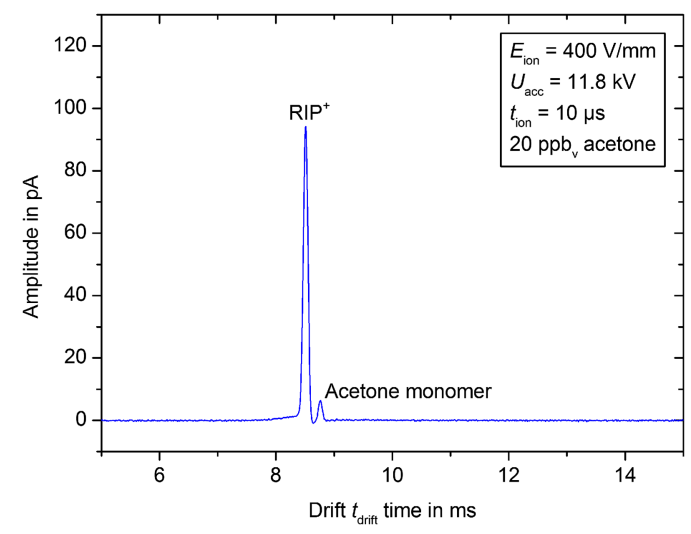

FIG. 9. Positive shutterless-IMS-spectrum of $20 \mathrm{ppb}_{\mathrm{v}}$ acetone in clean dry air. The drift voltage is set to $U_{\text {drift }}=5 \mathrm{kV}$. The total ion charge is controlled to a constant value of $Q_{\text {tot }}=9.9 \mathrm{fC}$. 
TABLE V. Limit of detection for acetone in $1 \mathrm{~s}$ at different electrical fields $E_{\text {ion }}$ and an ionization time of $10 \mu$ s. The total ion charge is controlled to the constant value at a RIP $^{+}$amplitude of $100 \mathrm{pA}$ without acetone. The comparable LoD for acetone in an IMS with field switching shutter (FS) $\left(t_{\text {ion }}=10 \mu \mathrm{s}, t_{\mathrm{FS}}\right.$ $=100 \mu \mathrm{s})$ is $\mathrm{LoD}_{\mathrm{FS}}=1 \mathrm{ppb}_{\mathrm{v}}$ at $R_{\mathrm{FS}}=92$.

\begin{tabular}{lccc}
\hline \hline$E_{\text {ion }}$ in V/mm & $U_{\text {acc,opt }}$ in $\mathrm{kV}$ & LoD in ppb & $R$ \\
\hline 100 & 10.6 & $1.8( \pm 1)$ & 75 \\
200 & 11.3 & $2.5( \pm 1)$ & 85 \\
400 & 11.8 & $3.5( \pm 1)$ & 89 \\
800 & 12.4 & $4.2( \pm 1)$ & 92 \\
\hline \hline
\end{tabular}

\section{CONCLUSION}

In this paper, we presented the realization and investigation of a shutterless IMS with a pulsed, nonradioactive electron source for initial ionization. With sufficiently short electron emission pulses with adjustable intensity, our nonradioactive electron source can generate small initial ion packets for a subsequent separation in the drift region. Therefore, a separated ion shutter mechanism is not required. For the investigation of the influence of different parameters on the analytical performance of this shutterless IMS, we first simulated the electron penetration depth into dry air for various electron acceleration voltages. With this result, we estimated the corresponding generated ion distribution in the ionization region. To generate an equal total ion charge at low electron acceleration voltages and hence at lower penetration depths, it is obvious that the ion density has to be drastically increased. This implies an increasing broadening influence by Coulombic repulsion. However, at high electron acceleration voltages, the penetration depth and thus the initial width of the ion packet is increased which implies high electrical fields in the ionization region. Both reduce the resolving power and therefore the analytical performance of this kind of IMS. By appropriate measurements, we found for each electrical field $E_{\text {ion }}$ in the ionization region an optimum acceleration voltage for the highest possible resolving power. However, for electrical fields above $E_{\text {ion }} \geq 800 \mathrm{~V} / \mathrm{mm}$ or ionization times below $t_{\text {ion }}$ $\leq 10 \mu \mathrm{s}$, no further significant improvement of the resolving power can be observed. Finally, the limit of detection for acetone was determined to $4.2 \mathrm{ppb}_{\mathrm{v}}$ at an electrical field of $E_{\text {ion }}=800 \mathrm{~V} / \mathrm{mm}$ and a resolving power of $R=92$. This can be improved down to $1.8 \mathrm{ppb}_{\mathrm{v}}$ by reducing the electrical field to $E_{\text {ion }}=100 \mathrm{~V} / \mathrm{mm}$ accepting a decreased resolving power of $R=75$. These limits of detection are comparable to a conventional IMS with identical ionization times. One particular advantage of this shutterless IMS is that a choice can be made between a better limit of detection and a higher resolving power, almost exclusively by varying the electrical field in the ionization region.

${ }^{1}$ G. A. Eiceman, Z. Karpas, and H. H. Hill, Ion Mobility Spectrometry, 3rd ed. (CRC Press, Boca Raton, 2013).

${ }^{2}$ A. T. Kirk and S. Zimmermann, "Bradbury-Nielsen vs. field switching shutters for high resolution drift tube ion mobility spectrometers," Int. J. Ion Mobility Spectrom. 17 (3-4), 131-137 (2014).

${ }^{3}$ J. I. Baumbach, S. Sielemann, Z. Xie, and H. Schmidt, "Detection of the gasoline components methyl tert -butyl ether, benzene, toluene, and $\mathrm{m}$-xylene using ion mobility spectrometers with a radioactive and UV ionization source," Anal. Chem. 75(6), 1483-1490 (2003).

${ }^{4}$ St. Sielemann, J. I. Baumbach, H. Schmidt, and P. Pilzecker, "Detection of alcohols using UV-ion mobility spetrometers," Anal. Chim. Acta 431(2), 293-301 (2001).

${ }^{5} \mathrm{H}$. Borsdorf, "Determination of $\mathrm{n}$-alkanes and branched chain alkanes by Corona discharge ion mobility spectrometry," Int. J. Ion Mobility Spectrom. 2, 9-14 (1999).

${ }^{6}$ C. L. Crawford and H. H. Hill, "Comparison of reactant and analyte ions for ${ }^{6} \mathrm{Nickel}$, corona discharge, and secondary electrospray ionization sources with ion mobility-mass spectrometry," Talanta 107, 225-232 (2013).

${ }^{7}$ G. A. Eiceman, J. H. Kremer, A. P. Snyder, and J. K. Tofferi, "Quantitative assessment of a corona discharge ion source in atmospheric pressure ionization-mass spectrometry for ambient air monitoring,” Int. J. Environ. Anal. Chem. 33 (3-4), 161-183 (1988).

${ }^{8}$ T. Reinecke, A. T. Kirk, A. Heptner, D. Niebuhr, S. Bottger, and S. Zimmermann, "A compact high-resolution X-ray ion mobility spectrometer," Rev. Sci. Instrum. 87(5), 53120 (2016).

${ }^{9}$ P. Cochems, A. T. Kirk, E. Bunert, M. Runge, P. Goncalves, and S. Zimmermann, "Fast pulsed operation of a small non-radioactive electron source with continuous emission current control,” Rev. Sci. Instrum. 86(6), 65102 (2015).

${ }^{10} \mathrm{P}$. Cochems, M. Runge, and S. Zimmermann, "A current controlled miniaturized non-radioactive electron emitter for atmospheric pressure chemical ionization based on thermionic emission," Sens. Actuators, A 206, 165-170 (2014).

${ }^{11}$ A. B. Kanu, M. M. Gribb, and H. H. Hill, "Predicting optimal resolving power for ambient pressure ion mobility spectrometry," Anal. Chem. 80(17), 6610-6619 (2008).

${ }^{12}$ F. T. Porter, "Beta decay energy of tritium," Phys. Rev. 115(2), 450-453 (1959).

${ }^{13}$ A. Morozov, T. Heindl, C. Skrobol, J. Wieser, R. Krücken, and A. Ulrich, "Transmission of $\sim 10 \mathrm{keV}$ electron beams through thin ceramic foils: Measurements and Monte Carlo simulations of electron energy distribution functions," Eur. Phys. J. D 48(3), 383-388 (2008).

${ }^{14}$ A. T. Kirk, M. Allers, P. Cochems, J. Langejuergen, and S. Zimmermann, "A compact high resolution ion mobility spectrometer for fast trace gas analysis," Analyst 138(18), 5200-5207 (2013).

${ }^{15}$ A. T. Kirk and S. Zimmermann, "An analytical model for the optimum drift voltage of drift tube ion mobility spectrometers with respect to resolving power and detection limits," Int. J. Ion Mobility Spectrom. 18 (3-4), 129-135 (2015).

${ }^{16}$ A. V. Tolmachev, B. H. Clowers, M. E. Belov, and R. D. Smith, "Coulombic effects in ion mobility spectrometry," Anal. Chem. 81(12), 4778-4787 (2009).

${ }^{17}$ W. F. Siems, C. Wu, E. E. Tarver, H. H. Hill, Jr., P. R. Larsen, and D. G. McMinn, "Measuring the resolving power of ion mobility spectrometers," Anal. Chem. 66(23), 4195-4201 (1994).

${ }^{18}$ H. E. Revercomb and E. A. Mason, "Theory of plasma chromatography/gaseous electrophoresis. Review," Anal. Chem. 47(7), 970-983 (1975). 